\title{
Characterization of ablated porcine bone and muscle using laser- induced acoustic wave method for tissue differentiation
}

\author{
Hervé K. Nguendon ${ }^{a^{*}}$, Neige Faivre ${ }^{\mathrm{b}}$, Bastian Meylan ${ }^{\mathrm{b}}$, Sergey Shevchik ${ }^{\mathrm{b}}$, Georg Rauter ${ }^{\mathrm{c}}$, Raphael \\ Guzman $^{\mathrm{d}}$, Philippe C. Cattin ${ }^{\mathrm{e}}$, Kilian Wasmer ${ }^{\mathrm{b}}$, Azhar Zam ${ }^{\mathrm{a}}$ \\ ${ }^{a}$ Biomedical Laser and Optics Group, Department of Biomedical Engineering, University of Basel, Gewerbestrasse. 14, Allschwil, Switzerland \\ ${ }^{b}$ Laboratory for Advanced Materials Processing, Empa-Swiss Federal Laboratories for Materials Science and Technology, 3602 Thun, Switzerland \\ ${ }^{c}$ Bio-Inspired RObots for MEDicine-Lab, Department of Biomedical Engineering, University of Basel, Gewerbestrasse. 14, Allschwil, Switzerland \\ ${ }^{d}$ Department of Neurosurgery, University Hospital Basel, Spitalstrasse 21, Basel, Switzerland \\ ${ }^{e}$ Center for medical Image Analysis and Navigation, Department of Biomedical Engineering, University of Basel, Gewerbestrasse. 14, Allschwil, \\ Switzerland
}

\begin{abstract}
A high power pulsed laser with millisecond pulse was used to interact with a bone and muscle of porcine, initiating an acoustic wave. We start to describe principle of laser ablation follows by the acoustic wave generation. Then, we present the characterization of these wave features for laser surgery applications.
\end{abstract}

Keywords: Acoustic signal, fiber Bragg Grating, laser ablation, shock wave, tissue differentiation

\section{INTRODUCTION}

Standard procedures in orthopedic surgery and tumor removals rely on mechanical tools such as saws or drills. These standard surgical tools induce severe mechanical vibrations resulting in heat formation that damages the surrounding tissues, leading to an impaired bone regeneration [1]. Avoiding this heat damage would improve the bone cutting characteristics a highly desired innovation in the field of maxillo-facial-, neuro- and orthopedic surgery. It is well-known that pulsed laser-ablation has significant advantages such as noncontact intervention, controlled bone excision, free-cut geometry and minimal invasiveness [1-3].

Numerous studies have investigated the fundamental processes that take place during laser ablation $[4,5]$. It has been found that the typical processes include optical absorption, electron heat transfer, and hydrodynamic ablation, which are accompanied by the generation of shock/acoustic signals that can be used to characterize the ablated material such as tissues [5]. Luckily, during ablations, lasers can create high wave amplitude signals with short dwell times or focused shock waves [6]. In fact, when processing material with a laser, acoustic waves are generated and have different origins such as expansion of the material, expansion of the plume/plasma, phase transformation, cracks formation and propagation.

It has also been understood that when laser interacts with matter, it can reflect, scatter, absorb or transmit depending upon the material characteristics (eq. physical, chemical, composition, and optical properties) and laser parameters (eq. laser energy, wavelength, spatial and temporal coherence, exposure time/pulse duration, etc.) are the key influential parameters in laser-matter interaction [7].

\footnotetext{
* Corresponding Author e-mail address: herve.nguendon@unibas.ch
} 
It is difficult to detect exactly when a laser has cut through the desired tissue and that there is the danger of cutting further in the underlying tissue $[2,8]$. This is particularly true if the laser system is not controlled by an in situ and realtime feedback control system that is able not only to differentiate specific types of human tissues but can automatically stop.

This paper presents acoustic wave characterizations for bone and muscle of porcine spare ribs ablated by a laser. In this work, we used a high power pulsed laser with $0.487 \mathrm{~ms}$ pulse durations to ablated porcine bones and muscles. We characterized the waves by comparing their peak amplitude, and their frequency shifts in the frequency domain. This information is a potential feature for tissue differentiations and can be stored as reference in the database of an optoacoustic feedback sensor system to automatically differentiate types of tissues during real-time laser surgery applications. The method has advantages to extract physical properties of tissues based on measured acoustic wave features.

\section{LASER-ACOUSTIC WAVES GENERATION}

The plasma ignition process includes bond breaking and plasma shielding during the laser pulse interaction with the material surface. Bond-breaking mechanisms influence the quantity and form of energy (kinetic, ionization and excitation) that atoms and ions can acquire. For nanosecond laser pulses of irradiance $<10^{8} \mathrm{~W} / \mathrm{cm}^{2}$, the dominant mechanism of plasma ignition is thermal vaporization [7], whereas for picosecond laser pulses with irradiance between $10^{8}$ and $10^{13} \mathrm{~W} / \mathrm{cm}^{2}$, both thermal and non-thermal mechanisms contribute to the plasma ignition. Nonetheless, with femtosecond laser pulses of irradiance $>10^{13} \mathrm{~W} / \mathrm{cm}^{2}$, the main bond-breaking (plasma ignition) mechanism is nonthermal, that is, Coulomb's explosion [7].

When high-power laser energy is absorbed, as high as $1 \mathrm{keV}$, the irradiated area rapidly reaches very high temperature,. This produces extremely rapid expansion of the surface material, and momentum conservation implies a strong disturbance, which travels into the solid material. The main bond-breaking (plasma ignition) mechanism is non-thermal, that is, Coulomb's explosion [7]. In our case, a millisecond pulsed laser has been used to ablate bone and muscle surrounded by air. The laser energy has been absorbed in a continuous fashion in the expanding plasma up to the surface for which the plasma wavelength is equal to the laser wavelength of $1.070 \mu \mathrm{m}$. In this state, one side of the ablation front is expanding away toward the laser and on the other side the specimen is being compressed and is moving in the opposite direction as illustrated as in Fig. 1.

The laser energy is absorbed in a continuous fashion in the expanding plasma up to the critical surface, defined as surface for which the plasma frequency is equal to the laser frequency. At the critical surface, the electron density is $n_{c}$ :

$$
n_{c}=\frac{\pi c^{2} m}{e^{2} \lambda^{2}}
$$

Where $m$ is the electron mass, $e$ its charge, and $\lambda$ the laser wavelength. The parameter $n_{c}$, is of fundamental importance in the study of laser-matter interactions. Energy from the absorption region is transported into the target by electron thermal conduction. In other words, on the one hand, the ablation front the material is expanding away from the target; on the other hand, the material is being compressed and is moving in the opposite direction. The compressed material generally forms a strong shock wave [5].

The target surface is instantly excited, ionized and the material vaporized into an extremely hot vapor plume also called plasma plume" with three main regions. (1) The core: near the target surface is the hottest and densest region (ionization state) of the plasma. (2) The mid-region: ions and neutrals (atoms + molecules) coexist due to the ongoing ionization and recombination process. (3) The outer region: is relatively cold and may absorb the radiation coming out of the core 
and mid-regions of the plasma. (4) The shock or acoustic wave front produced due to the explosive expansion of the plasma travels ahead of the plasma plume. Fig. 1 is the schematic of shock/acoustic wave emitting from laser ablation.

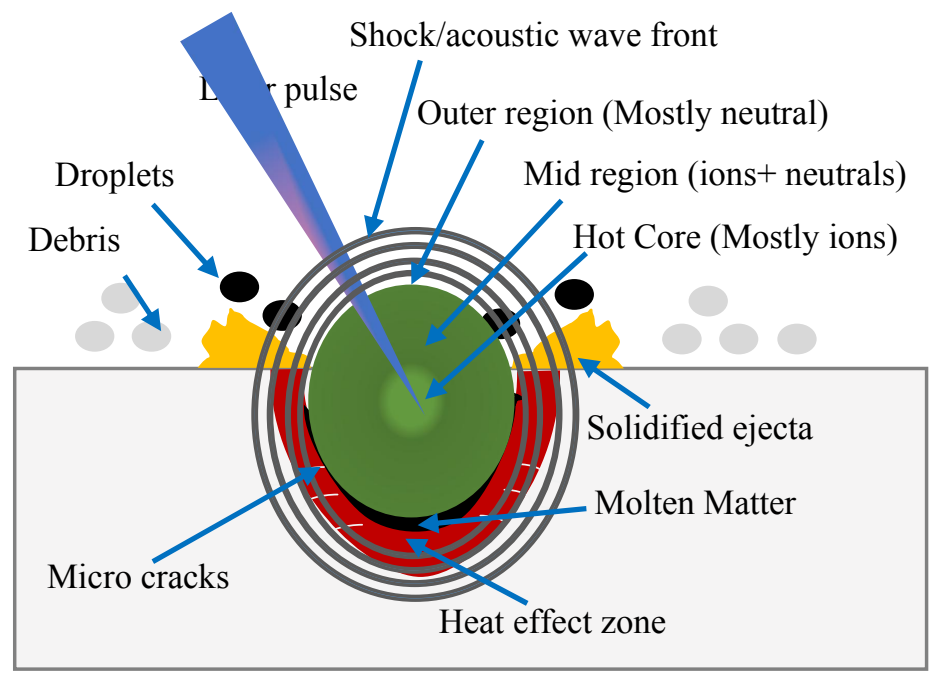

Figure 1. Schematic of laser induced plasma

For a typical Nd:YAG laser used in high power applications, with $\lambda=1.064 \mu \mathrm{m}, n_{e}=10^{21} \mathrm{~cm}^{-3}$. This is substantially less than solid density, typically on the order of $10^{23} \mathrm{~cm}^{-3}$, which has several implications. Thermal conduction between the critical and ablation regions is less efficient as this distance increases, or at a lower value of $n_{c}$. In addition, a variety of laser-plasma interactions can occur in the under-dense plasma $\left(n_{e}<n_{c}\right.$, ) which can generate highly energetic electrons which penetrate and "preheat" the sample. In general, higher shock pressures and less preheat over longer times are obtained with shorter wavelength laser irradiation; this is often obtained using harmonics of Nd-doped hosts [9].

The pressure obtained in this way can be described over wide ranges of intensity with a simple scaling relationship. A useful rule of thumb is:

$$
P \approx 40\left(\frac{I_{15}}{\lambda}\right)^{\frac{2}{3}}
$$

For pressure $P$ in Mbar (100 GPa), laser wavelength $\lambda$ in $\mu \mathrm{m}$, and intensity $I$ in units of $10^{15} \mathrm{~W} / \mathrm{cm}^{2}$. From this, we can see that an intensity of about $4.2 \times 10^{12} \mathrm{~W} / \mathrm{cm}^{2}$ will generate an ablation pressure of 1 Mbar. This is rather easily achieved with today's highly efficient lasers; for a spot diameter of $100 \mathrm{pm}$ and a pulse length of $2 \mathrm{~ns}$, a pulse energy of only about $0.7 \mathrm{~J}$ is needed [10].

However, the expansion of the material from the ablation front and through the critical surface can cause the distance between them to increase with time, lowering the pressure at the ablation front. Even a relatively constant intensity pulse will produce a decaying shock wave. After the laser decreases in intensity, the ablation pressure also decreases. These effects, as well as three-dimensional effects arising from the plasma expansion, lead to a strongly time-dependent ablation pressure. When the pressure falls, a rarefaction wave travels towards the shock front, eventually overtaking it. All these effects tend to produce a decreasing pressure at the shock front as it travels. It is, in principle, possible to overcome all but the last effect with appropriate temporal shaping of the laser pulse. A rather steady shock can be produced over a time of about equal to the half-width of the laser pulse [10]. This time dependence is in strong contrast to impact-generated shocks, which tend to be constant in amplitude over 1 microsecond intervals. It is to point out that side rarefactions are inevitable in experiments using small spatial scales, so that eventually the shock will become 
spherical and rapidly decreasing in amplitude. The spatial scales are also important, since laser spot sizes can be made as small as about $2 \lambda$, the diffraction limit. The effects of material expansion can be improved for low-intensity pulses $(\leq$ $10^{10} \mathrm{~W} / \mathrm{cm}^{2}$ ) by using a transparent material overlaying the sample which has a high optical damage threshold. For bone surgery, the best transition media for shock wave is water and gelatin because of the similarity in the acoustic impedance to the tissue $[2,11]$.

\section{SAMPLE PREPARATION}

Fresh pork spare ribs purchased from a local slaughterhouse was used for the laser-tissues ablation experiments. Scalpels were used to carefully separate the connective tissues from muscle and bone specimens as shown in Fig. 2.To obtain uniform sample sizes scalpels were also employed to peel out extra layers, maintaining the flat surface of the samples with a constant thickness difference at the surface of specimen of approximately $2 \mathrm{~mm}$. When samples were prepared, they were stored in distillate water at $4^{\circ} \mathrm{C}$ for $12 \mathrm{~h}$ prior to experiment, aiming at a uniform hydration state over all the tissue samples. Using, an electronic calliper, the measured bone and muscle thicknesses were $10.80 \mathrm{~mm}$ and $11.00 \mathrm{~mm}$, respectively.
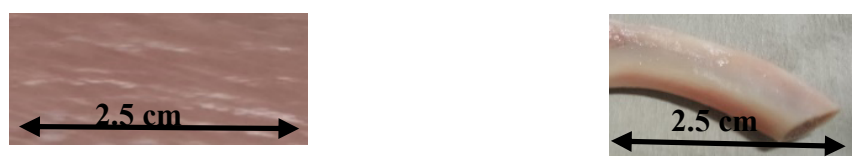

Figure 2. a (left) Muscle Sample, and b (Right) Bone Sample

\section{EXPERIMENTAL SETUP}

Fig. 3 gives a schematic view of the experimental setup using a fiber Bragg grating (FBG) to measure the acoustic waves. For a precise cutting of the bones and muscles, we used a fiber source StarFiber 150 P (ROFIN-LASEG LASER) with a $1070 \mathrm{~nm}$ wavelength with a scanner head from Scanlab (hurrySCAN). The laser beam was transmitted through a single-mode optical fiber with a $12 \mu \mathrm{m}$ core diameter and focused at the surface of the sample with a focal distance of $150 \mathrm{~mm}$. This setup provides a spot size diameter of about $30 \mu \mathrm{m}$ at the focal point $\left(2 w_{0}\right)$. The pulse duration was 0.487 ms with a pulse energy of $750 \mathrm{~mJ}$ so that the energy fluence delivered by a single pulse on the sample surface (bone or muscle) was $265.26 \mathrm{~J} / \mathrm{mm}^{2}$. More details of the setup can be found in [12].

The emitted acoustic wave was simultaneously measured with a microphone (PAC AM41 SNAA05) and a FBG system (e: FFT Corning SMF-28) with a first grating position at $1 \mathrm{~mm}$, a FBG length of $3.1 \mathrm{~nm}$ and a centre wavelength of $1547.09 \mathrm{~nm}$ at a $3 \mathrm{~dB}$ bandwidth of $0.497 \mathrm{~nm}$ and a reflectivity of $57 \%$. The FBG had a core diameter of $9 \mu \mathrm{m}$ with a cladding diameter of $125 \mu \mathrm{m}$. Both sensors are situated within $2 \mathrm{~cm}$ from the ablation spot. This setup was focussed at measuring the acoustic waves with the FBG.

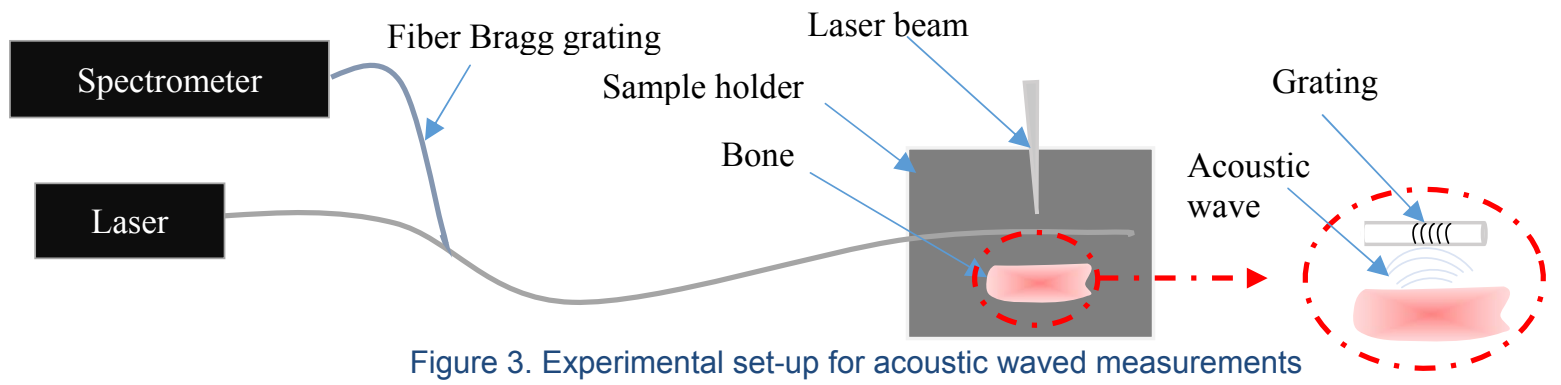




\section{RESULTS}

\subsection{Baseline Measurements}

During the initialization phase, we measured the light from the FBG without any ablation. We collected ten signals from the photodiode under the same condition. Figure $4 \mathrm{a}$ are respectively, the corresponding time (top) and frequency (bottom) domains. Ten measurements of the FBG without any ablation show variations in the amplitudes in the time domain. In the frequency domain, it is also visible that each measured data is not always the same. Table 1 resumes the resonant frequencies of each baseline measurement within 0.0095-0.0210 $\mathrm{MHz}$. To enhance the signal, we took the average of the ten measurements as shown in Figure $4 \mathrm{~b}$. The average resonant frequency and standard deviation are respectively $0.0095 \pm 0.0060 \mathrm{MHz}$. Indeed, averaging the signals allow to reduce the random signals and thus improve the results. Having the baseline measurements, ten experimental results for the acoustic waves during ablated bone and muscle have been compared.
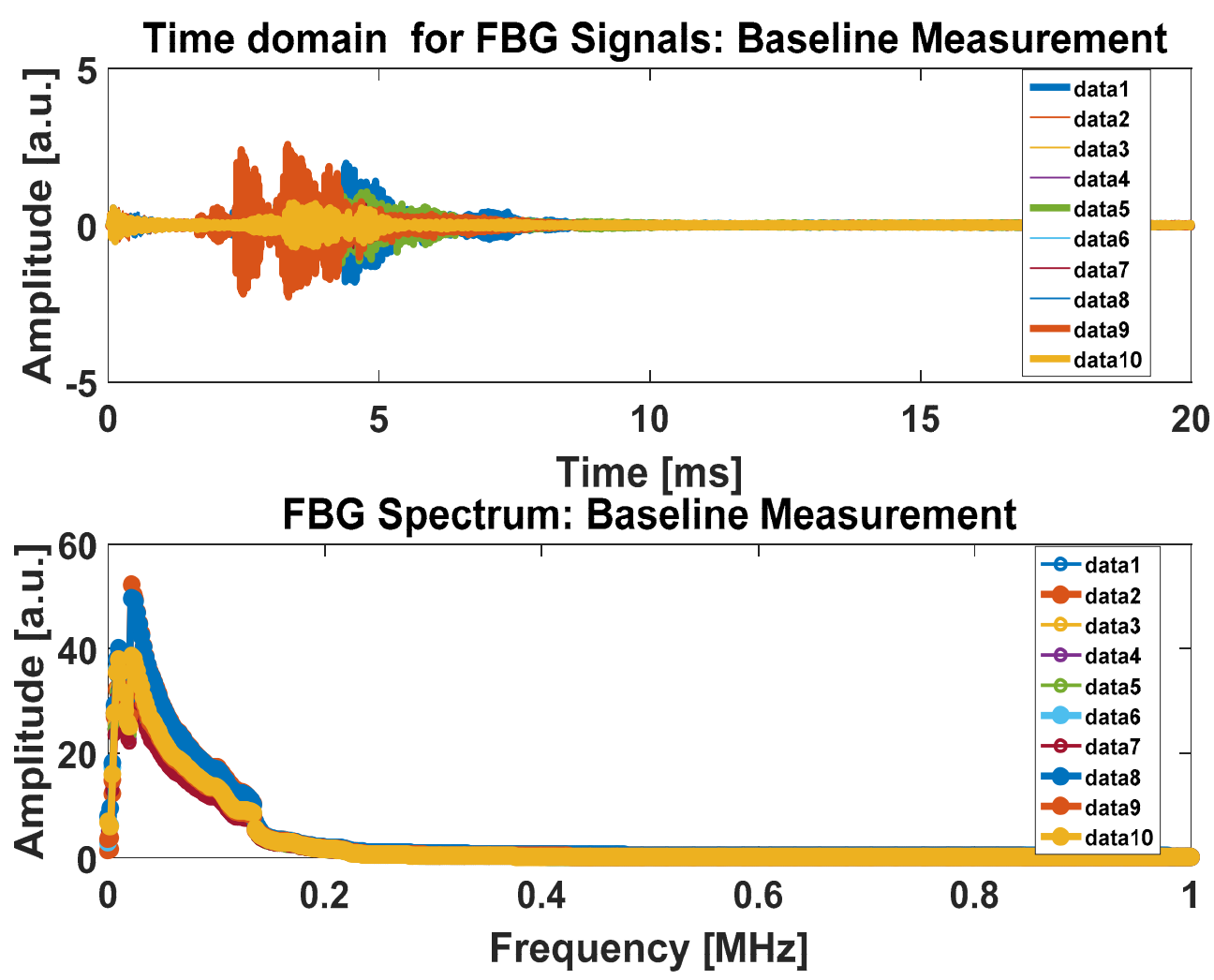

Figure 4a. Baseline measurements in time- and frequency-domain from Fiber Bragg Grating 

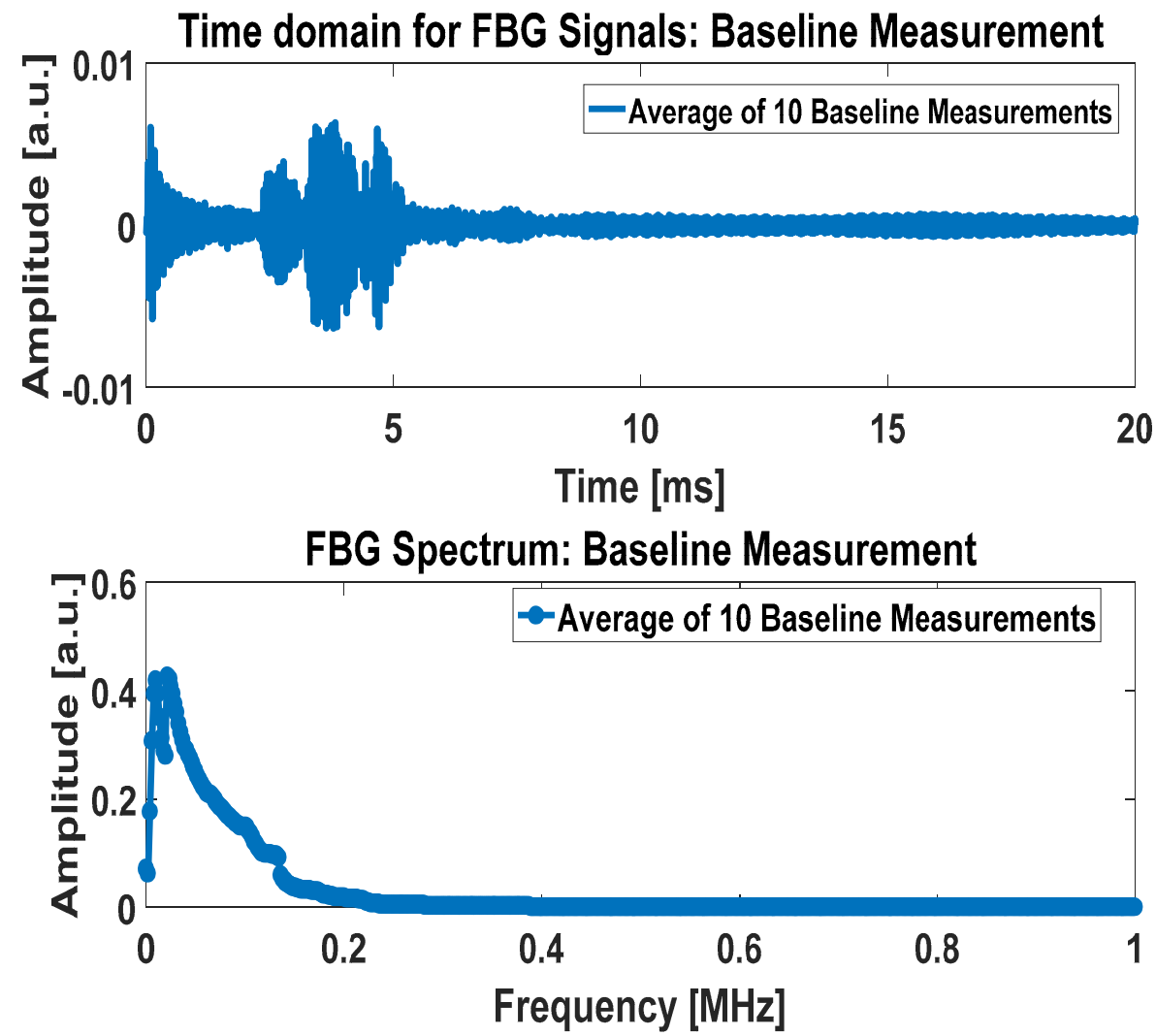

Figure 4b. Average of ten measurements of Baseline measured signal in time- and frequency-domain from Fiber Bragg Grating

\begin{tabular}{|c|c|c|c|c|c|c|c|c|c|c|}
\hline Ablated positions & $\begin{array}{l}\text { Position } \\
1\end{array}$ & $\begin{array}{l}\text { Position } \\
1\end{array}$ & $\begin{array}{l}\text { Position } \\
1\end{array}$ & $\begin{array}{l}\text { Position } \\
1\end{array}$ & $\begin{array}{l}\text { Position } \\
1\end{array}$ & $\begin{array}{l}\text { Position } \\
1\end{array}$ & $\begin{array}{l}\text { Position } \\
1\end{array}$ & $\begin{array}{l}\text { Position } \\
1\end{array}$ & $\begin{array}{l}\text { Position } \\
1\end{array}$ & Position 1 \\
\hline Resonant Frequency(MHz) & 0.0210 & 0.0210 & 0.0210 & 0.0095 & 0.0095 & 0.0095 & 0.0095 & 0.0210 & 0.0095 & 0.0210 \\
\hline Standard Deviation & \multicolumn{10}{|c|}{0.0060} \\
\hline
\end{tabular}

\subsection{Acoustic Wave Measurements}

We focused the laser beam at the same position at the surface of the specimens and we sent ten pulses with a repetition rate of $1 \mathrm{~Hz}$. Figure $5 \mathrm{a}$ and $5 \mathrm{~b}$ show the ten acoustic signals acquired by the FBG in the time domain during laser ablation of the muscle and bone, respectively. The peak-to-peak amplitudes measured by the FBG for the muscle 
specimen are higher than the ones generated by the bone. This was expected because an acoustic wave generated by the bone has higher amplitude and therefore a higher pressure than the one for muscle.

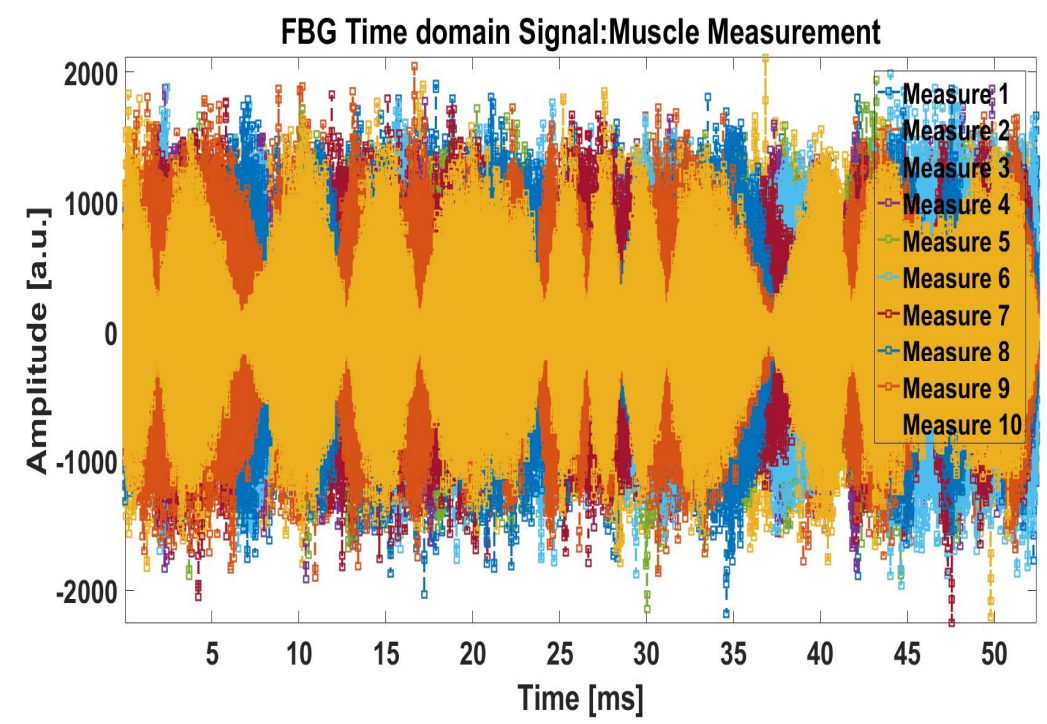

Figure 5a. Fiber Bragg Grating (FBG) Signal Measurements in the time domain for ten emitted pulses to the muscle sample

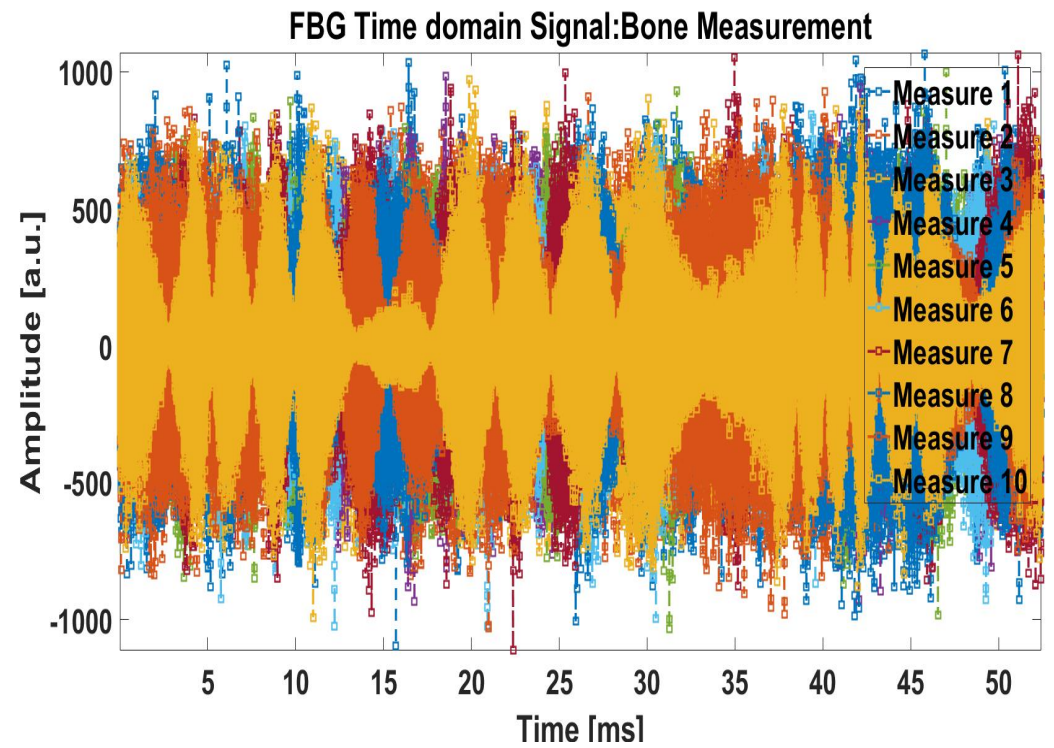

Figure 5b. Fiber Bragg Grating (FBG) Signal Measurements in the time domain for ten emitted pulses to the bone sample 
The corresponding frequency domain of the ten acoustic waves of both muscle and bone are depicted in Figure 6a and $b$. The resonant frequencies for muscle is in the range of $0.1389-0.2778 \mathrm{MHz}$ with a standard deviation of $0.0732 \mathrm{MHz}$ and for bone within $0.1389-0.5556 \mathrm{MHz}$ with a standard deviation of $0.1610 \mathrm{MHz}$. In order to improve the results, the average frequencies of each signal have been post-processed. Accordingly, the average resonant frequency of the ten signals from the muscular tissue appears at $0.139 \mathrm{MHz}$. In contrast, the baseline peak and the signal related to bony tissue has a resonant frequency at $0.417 \mathrm{MHz}$ (Fig. 7). Thus, we observed a shift of $0.278 \mathrm{MHz}$ in the maximum peak in the frequency domain between the muscle and the bone tissue. At the same time, also the signal amplitudes provide clearly distinctive information between bony and muscular tissue: muscular tissue reveals a maximum peak amplitude of $40.000 \mathrm{mV}$ whereas bone rises up to $58.000 \mathrm{mV}$. An additional discriminative features is the maximum peak amplitude frequency which is higher for muscle than for bone. All this information could be used in future to differentiate bony from muscular tissues in real-time during laser surgery. However, the authors believe that a state-of-the-art signal processing approach such as machine learning to classify the acoustic features could provide an alternative more robust method for discriminating between the different tissues.

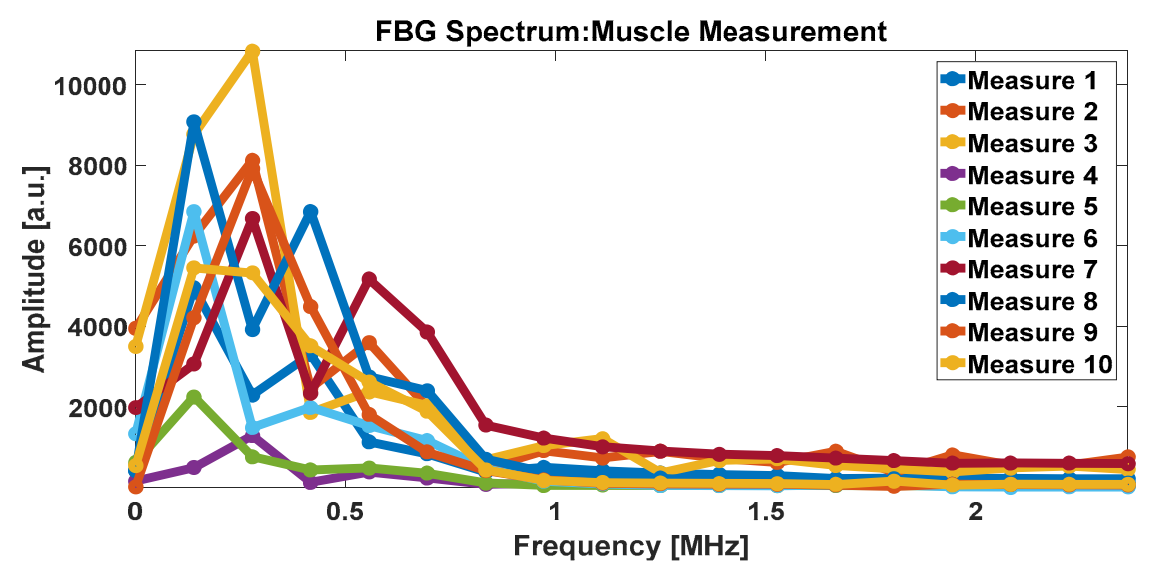

Figure 6a. Fiber Bragg Grating (FBG) Signal Measurements in the in frequency domain for ten emitted pulse to the muscle sample

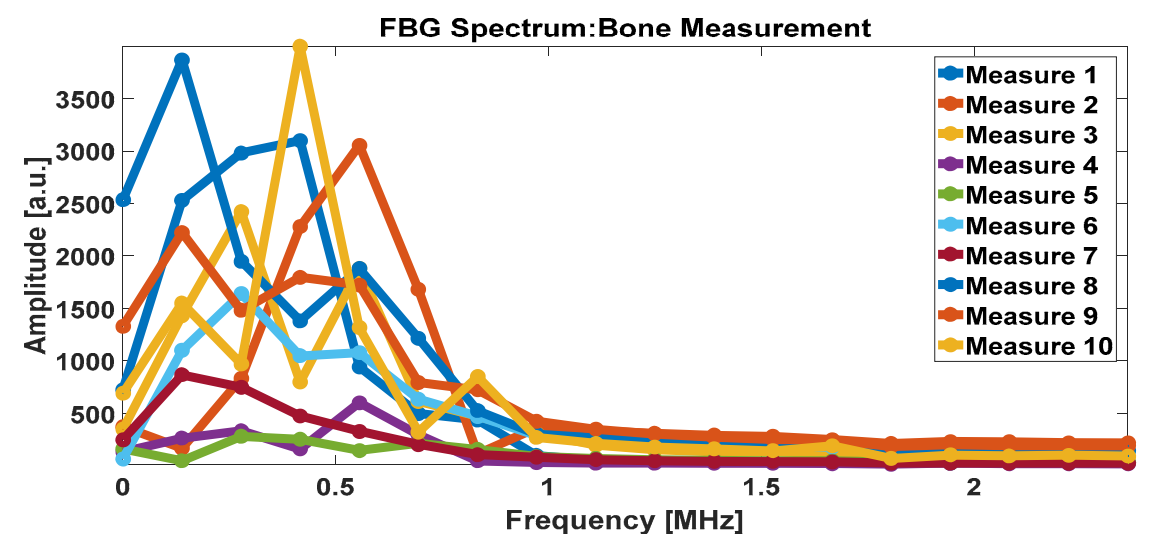

Figure 6b. Fiber Bragg Grating (FBG) Signal Measurements in the frequency domain for ten emitted pulse to the bone sample 


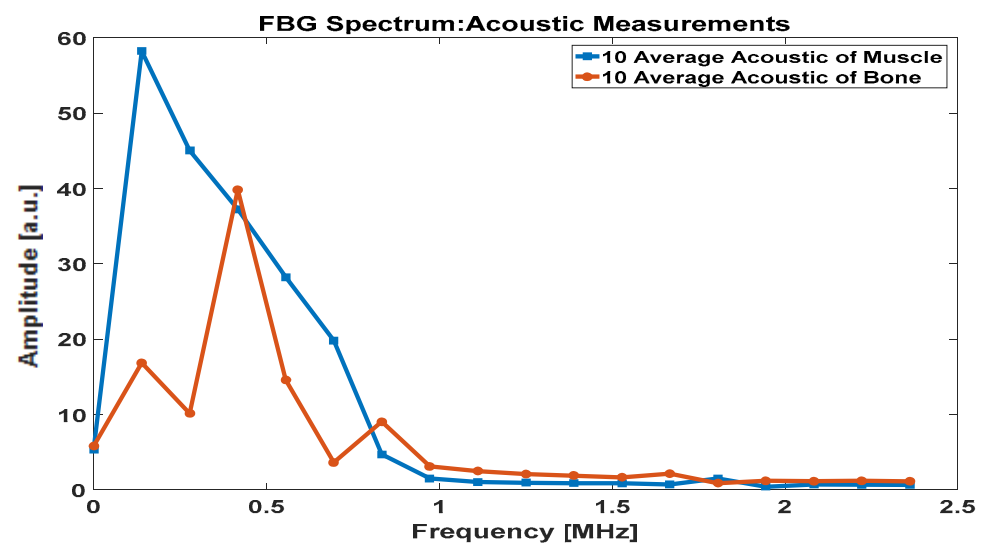

Figure 7. Ten average of Fiber Bragg Grating (FBG) Signal Measurements in frequency-domain when the FBG is stressed by the acoustic wave of Muscle and Bone

\section{CONCLUSION}

We presented preliminary results of acoustic signals acquired during the laser cutting of bone and muscle using Fiber Bragg Grating (FBG) as a sensor. It is found that the generated signal from laser-muscle ablation has a peak amplitude frequency higher than the one of bone. The average response to the acoustic waves using ten acoustic waves generated at the same ablated position for bone and muscles exhibited the resonant frequency at $0.417 \mathrm{MHz}$ and $0.139 \mathrm{MHz}$ respectively. This acoustic feature can be used for tissue differentiation. Therefore, the information needs to be stored as reference in a database of opto-acoustic feedback sensor systems to automatically differentiate tissues during real-time laser surgery applications. We will conduct more experiments under varying conditions like different specimens, different distances, and spray. Furthermore, we will investigate the use of machine learning techniques for better differentiation.

\section{ACKNOWLEDGEMENTS}

The authors gratefully acknowledge funding of the Werner Siemens Foundation through the MIRACLE (short for Minimally Invasive Robot-Assisted Computer-guided LaserosteotomE) project.

\section{REFERENCES}

[1] K.w. Baek, W. Deibel, D. Marinov, M. Griessen, M. Dard, A. Bruno, H.F. Zeilhofer, P. Cattin, P. Juergens, A comparative investigation of bone surface after cutting with mechanical tools and Er: YAG laser, Lasers Surg Med. 47(5), 426-432 (2015).

[2] K. Hyun Wook, L. Ho, C. Shaochen, A.J. Welch, Enhancement of bovine bone ablation assisted by a transparent liquid Layer on a target surface, IEEE J. Quant. Electron, 42 (7), 633 - 642 (2006) 
[3] Z.Z. Li, L. Reinisch, W.P. Van de Merwe, Bone ablation with Er: YAG and CO2 laser: study of thermal and acoustic effects, Lasers Surg Med. 12(1), 79-85 (1992)

[4] A. Vogel, V. Venugopalan, Mechanisms of pulsed laser ablation of biological tissues, Chem Rev. 103(2), 577-644 (2003).

[5] W. Bai, G.J. Diebold, C.M. Wynn, R.W. Haupt, J.H. Doherty, A numerical study of shock waves generated through laser ablation of explosives, J. Appl. Phys. 120, 194903 (2016)

[6] M. Boustie, T. Berthe, T. De Rességuier, M. Arrigoni, Laser Shock Waves: Fundamentals and Applications, in: 1st international symposium on Laser Ultrasonics, Montreal, France, 2008.

[7] K. Chaudhary, S.Z.H. Rizvi, J. Ali, Laser-Induced Plasma and its Applications, InTech, 259-291 (2016).

[8] M.H. Niemz, Laser-tissue interactions: fundamentals and applications, Springer Science \& Business Media, Medical,135-305 (2013).

[9] A. Ng, D. Parfeniuk, L. Da Silva, P. Celliers, Laser-driven shock wave experiments at the University of the British Columbia, Laser and Particle Beams (1986).

[10] Laser-generated Shock Waves and Applications to Advanced Materials, Lawrence Livermore National Laboratory, 1996.

[11] S.K. Shrivastava, Kailash, Shock wave treatment in medicine, J. Biosci. 30(2), 269-75 (2005).

[12] F. Vakili-Farahani, J. Lungershausen, K. Wasmer, Process Parameter Optimization for Wobbling Laser Spot Welding of Ti6Al4V Alloy, Physics Procedia, 83, 283-493 (2016). 